\title{
Visualization of Traveling Vortices in the Boundary Layer on a Rotating Disk under Orbital Motion
}

\author{
Mizue Munekata, Keiichiro Kubo, Hiroyuki Yoshikawa \\ Department of Mechanical Systems Engineering, Kumamoto University, Kumamoto, Japan \\ Email: munekata@gpo.kumamoto-u.ac.jp
}

Received 22 January 2015; accepted 12 February 2015; published 15 February 2015

Copyright (C) 2015 by authors and Scientific Research Publishing Inc.

This work is licensed under the Creative Commons Attribution International License (CC BY). http://creativecommons.org/licenses/by/4.0/

(c) (i) Open Access

\begin{abstract}
The objective of this study is to experimentally visualize traveling vortices in the boundary layer on a rotating disk under orbital motion. The orbital radius is half of the disk's diameter $(200 \mathrm{~mm})$ and the maximum speed of orbital motion is $\mathbf{5 0 0}$ revolutions per minute. The Reynolds number in the pure-rotation case is $2.77 \times 10^{5}$. The characteristics of two types of traveling vortices are visualized by a smoke-wire method. The first type is transition vortices. In the pure-rotation case, they arise at circumferentially equal intervals, and are not traveling but stationary relative to the rotational disk. The result of visualization of this type shows that the intervals between transient vortices change in a circumferential direction, or in an orbital radial direction, on the rotating disk under orbital motion. The second type is new arc-shaped vortices that correspond to low-frequency disturbances. As orbital speed increases, the radial traveling velocities of the low-frequency disturbances increase and the intervals between low-frequency disturbances decrease.
\end{abstract}

\section{Keywords}

Rotating Disk, Orbital Motion, Boundary Transition, Flow Visualization, Hot-Wire

\section{Introduction}

The boundary layer on a rotating disk under orbital motion has been scarcely studied by other researchers. In contrast, the boundary layer on a disk under rotation alone has been investigated in many studies [1]-[3]. Exact solutions of Navier-Stokes equations for the laminar boundary layer flow on a rotating disk were obtained theoretically by von Karman [4] [5]. When the rotational speed of a disk increases and the disk diameter is large, the Reynolds number of the boundary layer (which depends on the local radius, disk rotation speed, and kinematic 
viscosity of the fluid) will exceed the critical value. When this happens, a boundary layer transition will occur and then about 30 transient vortices will be formed in the outer portion of the laminar region on the rotating disk. Gregory [2], Kohama [3], Kobayashi [6] and others have experimentally visualized these transient vortices. From those results, the critical Reynolds number of the boundary layer transition was estimated by stability analysis. Kohama [3] summarized the results obtained by many researchers for boundary layer transitions on rotating disks.

Transient vortices generated on a disk under rotation alone will prevent formation of a film with uniform thickness during the spin-coating process of semiconductor manufacturing. Because the generated vortices are stationary relative to the disk, they will cause streaks to form on the film. To resolve this problem, we have refined our original technique, which uses orbital motion during spin coating to prevent streaking.

In our earlier work [7] [8], we found that when the orbital speed was increased, the laminar region narrowed and the transition point moved inward on the disk. This occurs independently of the direction of rotation. Moreover, we examined the characteristics of transient vortices and low-frequency disturbances by finding the power spectra of velocity fluctuation in the boundary layer on a rotating disk under orbital motion. From the velocity fluctuation spectra, we expect that both types of traveling vortices-transient vortices and low-frequency disturbances - travel on the disk. However, the dynamic behavior of the vortices on the disk is not clear. The objective of the present study is to experimentally visualize the two types of traveling vortices in the boundary layer on a disk undergoing both rotation and orbital motion. The first type is transition vortices, which are stationary vortices that arise in the pure-rotation case. The second type is low-frequency disturbances. The characteristics of both traveling vortices are visualized by a smoke-wire method. The traveling velocities and the intervals between low-frequency disturbances are measured by using two hot-wire probes.

\section{Experimental Procedure}

\subsection{Experimental Apparatus}

The experimental apparatus (Figure 1(a)) consists of a rotating disk mounted on an orbital rotation base, which carries the disk along its orbit in a way analogous to the orbit of a planet around the sun. Here, the orbital radius $R_{0}$ is $100 \mathrm{~mm}(=R)$, the disk radius $R$ is $100 \mathrm{~mm}$, and the disk edge thickness is $1 \mathrm{~mm}$. The disk is installed on an orbital rotation base. The complete details of the experimental apparatus are described in our previous report [7]. The counterclockwise direction is taken as the positive direction for both rotation and revolution (i.e., orbital movement). Trials are performed at a rotational speed $N$ of $+4000 \mathrm{rpm}$ and orbital speeds $N_{\mathrm{o}}$ in the range from -500 to $+500 \mathrm{rpm}$ (inclusive). The Reynolds number in the pure-rotation case is $2.77 \times 10^{5}$. A boundary layer transition occurs on the disk in this Reynolds number. The critical Reynolds number of the boundary layer transition is $8.2 \times 10^{4}$ according to stability analysis [9].

Two distinct radial coordinate systems (Figure 1(b)) are used: a fixed system and a moving system. The origin of the fixed coordinate system is the center of orbit, and the parameter showing distance in the radial direction from this center is denoted $r_{\mathrm{o}}$. The origin of the moving coordinate system is the center of rotation. In the moving coordinate system, the coordinates are parameterized as $r, \theta$, and $z$ for distance in the radial direction, angle in the circumferential direction, and distance in the axial direction, respectively. For points inside of the orbital radius $\left(r_{\mathrm{o}}<R_{\mathrm{o}}\right)$, the radial distance $r$ in the moving coordinate system will be represented by a negative number.

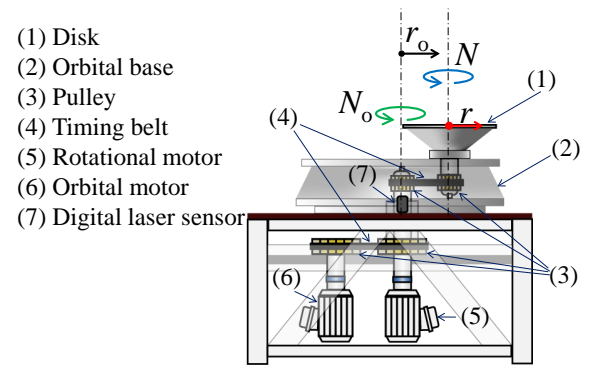

(a)

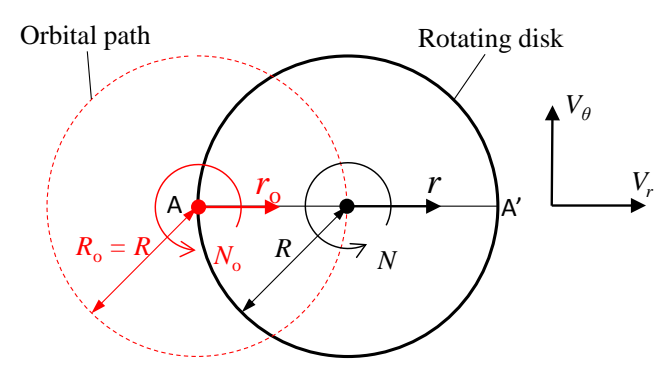

(b)

Figure 1. Schematic views of experimental apparatus. (a) Experimental apparatus; (b) Coordinate systems. 


\subsection{Hot-Wire Measurement}

The velocity field of the boundary layer flow on the disk is measured with a single hot-wire anemometer at a fixed angular position by using a timing-mark laser sensor. The hot-wire is positioned parallel to the disk surface and aligned normal to rotation when measuring the tangential (i.e., circumferential) velocity component $V_{\theta}$. An analog-to-digital converter is triggered by a timing pulse signal from the orbital base; this ensures that the center of each time record represents the same orbital angular position. Velocity data for 1024 points are sampled at every trigger signal for at most 750 orbital revolutions at a sampling frequency of $100 \mathrm{kHz}$ (low-pass filter: 12 $\mathrm{kHz})$.

The velocity fluctuation data of $V_{\theta}^{*}$ are analyzed by the fast Fourier transform method every 1024 points, and then the ensemble-averaged power spectral density (PSD) is calculated. The radial boundary layer is measured at a height $z=0.47-0.48 \mathrm{~mm}$ from the disk's surface (so that $z / \delta=2.5$ ).

The radial propagation velocity and the interval between low frequency disturbances were measured by using two hot-wire probes placed $5 \mathrm{~mm}$ apart at $z / R=+0.9$. One probe measures in the orbital direction and the other measures in the rotational direction.

\subsection{Flow Visualization}

The boundary layer flow is visualized by the smoke-wire method using the system shown in Figure 2. Nichrome wires of $50 \mu \mathrm{m}$ in diameter are set over the rotation disk with a gap of $0.5-0.6 \mathrm{~mm}$ between the wire and the disk. The nichrome wires are coated in liquid paraffin. High voltage is applied to these wires, which generates smoke. The flow of the smoke is captured from two viewpoints.

For the first viewpoint, an argon ion laser sheet is inserted parallel to the disk so as to capture a top view of the smoke flow. This top view is captured from above the disk by a fixed high-speed camera. For the second viewpoint, the argon ion laser sheet is inserted normal to rotation so as to capture the low-frequency disturbances. The smoke flow is captured from the side of the disk by the high-speed camera.

\section{Results and Discussion}

\subsection{Effects on Velocity Field of Orbital Motion}

The profiles of the mean tangential velocity component $\left(V_{\theta}\right)$ in the orbit radial direction are shown in Figure 3 and Figure 4. Both figures are obtained in our previous studies [7] [8]. The solid lines in the figures indicate the theoretical value of the mean for each velocity component in the laminar boundary layer on the purely rotating disk, as reported by von Karman [4] [5]. The velocities in the laminar region are distributed linearly. The laminar region has previously been shown to narrow as the orbital velocity increases [3] [6].

In Figure 3, the direction of rotation of the disk is opposite to the direction of orbital motion $(N=+4000 \mathrm{rpm}$, $\left.N_{\mathrm{o}}<0\right)$. At the outward region from the spiral flow's center $\left(V_{\theta}=0\right)$, which is located outward in the orbital

(1) High-speed camera

(2) Nichrome wire

(3) Disk

(4) Orbital base

(5) Argon ion laser sheet

(6) PC

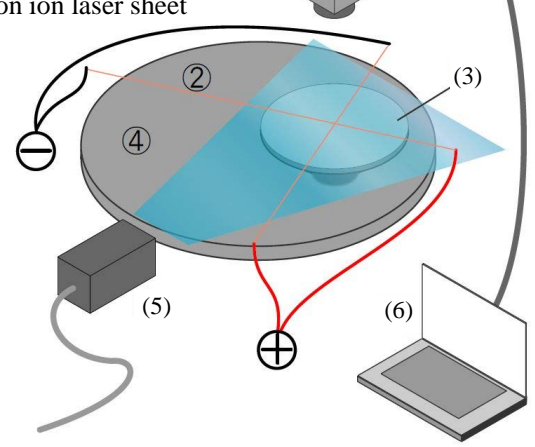

Figure 2. Flow visualization system by the smoke-wire method. 


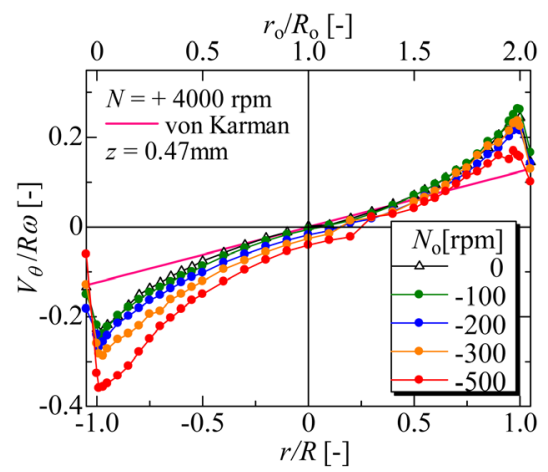

Figure 3. Mean tangential velocity distributions in the radial direction $(N=+4000 \mathrm{rpm}$, opposite rotational and orbital directions).

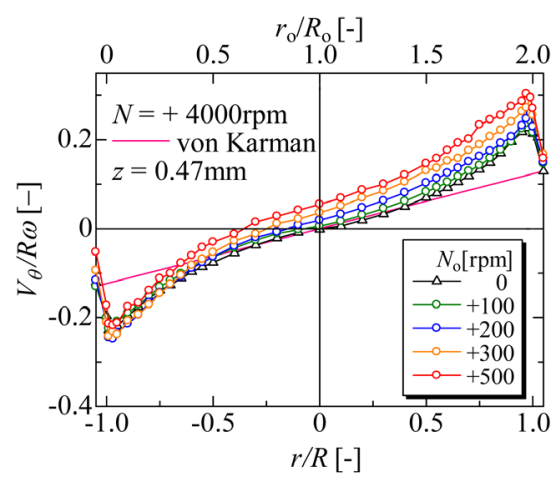

Figure 4. Mean tangential velocity distributions in the radial direction $(N=+4000 \mathrm{rpm}$, coinciding rotational and orbital directions).

radial direction, the mean tangential velocity decreases as the orbital velocity increases. This region shows decelerated tangential velocity on the disk's surface due to the orbital motion being in the direction opposite to the direction of disk rotation. In contrast, at the inward region from the spiral's center, there is an accelerated region, where the mean tangential velocity increases as the orbital velocity increases.

When the directions of rotation and orbital motion are the same (at $N=+4000 \mathrm{rpm}, N_{\mathrm{o}}>0$ ), the mean tangential velocity increases at the outward region from the spiral's center as the orbital velocity increases (Figure 4). At the inward region from the spiral's center, the tangential velocity in the laminar region as the orbital velocity increases.

\subsection{Visualization of Behavior of Transition Vortices}

Contour maps [8] of the ensemble-averaged PSD of $V_{\theta}^{*}$ fluctuation and the top-view visualization images are shown in Figures 5-9. The vertical axis in panel (a) of each figure indicates the radial location on the disk. The tops of the disk images in panel (b) of each figure indicate the location of $z / R=+0.1$, and the bottoms of the disk images indicate the location of $z / R=-0.1$.

Figure 5 shows the pure-rotation case $(N=+4000 \mathrm{rpm})$. The transient vortices, of which there are 29 , are shown in the outer portion on the disk in Figure 5(b). The high PSD region around $1.9 \mathrm{kHz}$ is distributed at the outer portion on the disk, where $|r| / R>0.75$. The velocity fluctuations at high levels of PSD correspond to oscillation due to the transient vortices, as shown in Figure 5(b). It is shown in Figure 5(b) that the transient vortices generated at the outer region of the disk occur at circumferentially equal intervals.

Figure 6 shows the case of rotational velocity $N=+4000 \mathrm{rpm}$ and orbital velocity $N_{\mathrm{o}}=-100 \mathrm{rpm}$. At the outer region of the disk, where $r / R>+0.75\left(r_{\mathrm{o}} / R_{\mathrm{o}}>1.75\right)$, the frequency band with high PSD due to the transient vortices shifts to a low-frequency band (in comparison with the corresponding frequency band from the pure- 


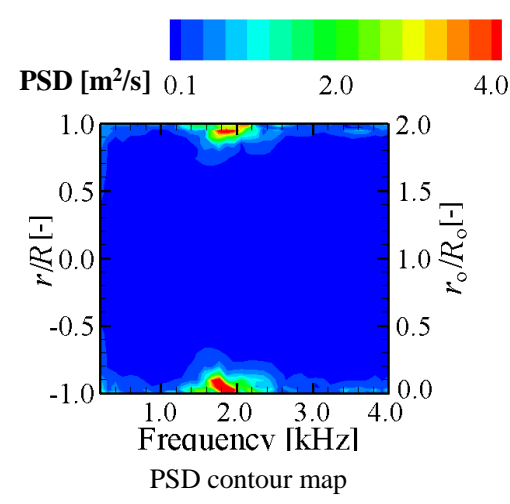

(a)

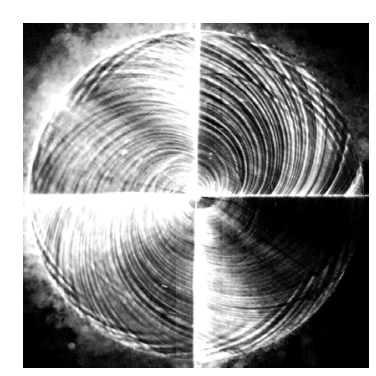

Visualization image

(b)

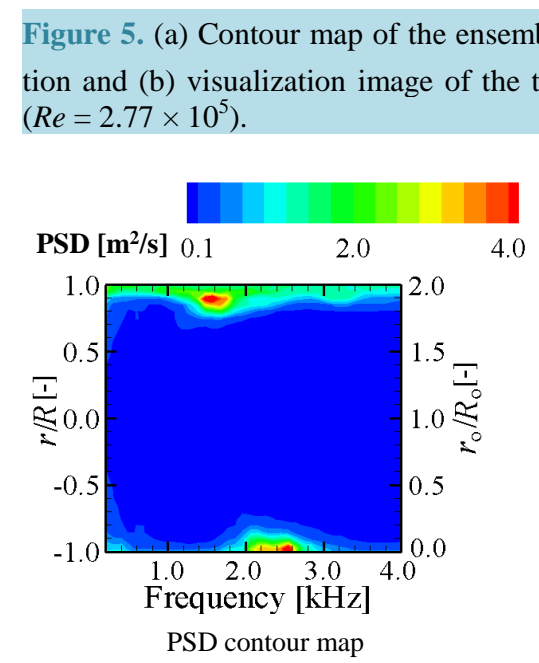

(a)

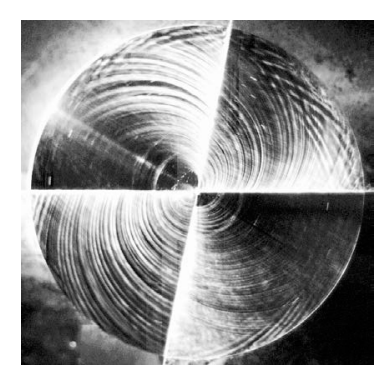

Visualization image

(b)

\footnotetext{
Figure 6. (a) Contour map of the ensemble-averaged PSD of $V_{\theta}^{*}$ fluctuation and (b) visualization image of the top view with $N=+4000 \mathrm{rpm}, N_{\mathrm{o}}=$ $-100 \mathrm{rpm}$.
}

rotation case) because the tangential velocities are decreased. At $r / R<-0.75 \quad\left(r_{0} / R_{0}>0.25\right)$ in the region where the tangential velocities increase, the frequency band with high PSD due to transient vortices shifts to a high-frequency band (in comparison with the corresponding frequency band from the pure-rotation case). These frequency shifts are considerably larger than the Doppler shifts induced by the addition of orbital motion. Figure 6(b) provides evidence that the interval between the transient vortices become wider at $r / R>+0.75$ $\left(r_{0} / R_{0}>1.75\right)$ and narrower at $r / R<-0.75\left(r_{0} / R_{0}>0.25\right)$. That is, the visualization images validate the predictions about the behavior of the transient vortices under the orbital motion, which were made from the PSD contour map of $V_{\theta}^{*}$ fluctuation. These vortices are not stationary relative to the rotational disk, and the intervals between them become narrower in the increased-velocity region and become wider in the decreased-velocity region.

\subsection{Visualization of Traveling Vortices Caused by Low-Frequency Disturbances}

Figure 7 and Figure 8 show the case of rotational velocity $N=+4000 \mathrm{rpm}$ and orbital velocity $N_{\mathrm{o}}= \pm 200 \mathrm{rpm}$. The shift of frequency bands due to the transient vortices at $N_{\mathrm{o}}= \pm 200 \mathrm{rpm}$ is more pronounced than in the case of $N_{\mathrm{o}}= \pm 100 \mathrm{rpm}$; however, the values of PSD are decreased considerably. The transient vortices at $N_{\mathrm{o}}= \pm 200$ rpm are not observed in Figure 7(b) and Figure 8(b) because the wave due to the transient vortices is generated only intermittently [8], and most of the smoke flows into the new vortices generated above the transient vortices.

The new vortices are observed at the outer region of the disk $(|r| / R>0.5)$ in Figure 7(b) and Figure 8(b). 


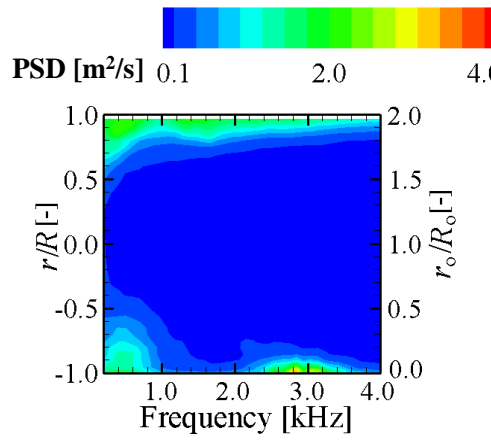

PSD contour map

(a)

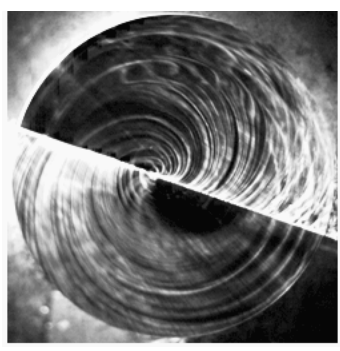

Visualization image

(b)

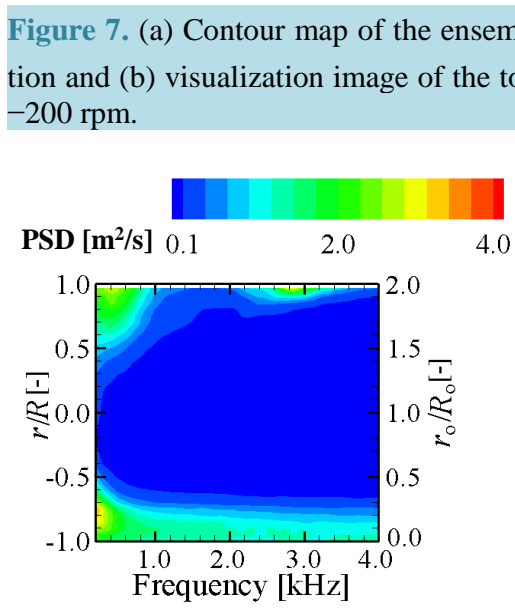

PSD contour map

(a)

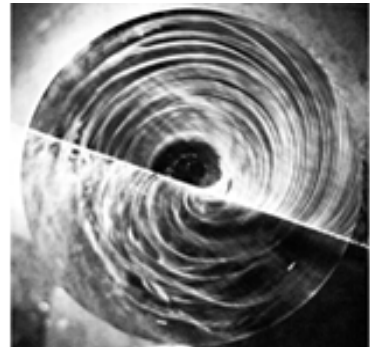

Visualization image

(b)

Figure 8. (a) Contour map of the ensemble-averaged PSD of $V_{\theta}^{*}$ fluctuation and (b) visualization image of the top view at $N=+4000 \mathrm{rpm}, N_{\mathrm{o}}=$ $+200 \mathrm{rpm}$.

The smoke streaks caused by the vortices are arc-shaped. These arc-shaped streaks are formed at roughly regular intervals and move in the radially outward direction on the disk, which was determined from the visualization movies.

Figure 9 and Figure 10 show the cases with rotational velocity $N=+4000 \mathrm{rpm}$ and orbital velocity $N_{\mathrm{o}}= \pm 300$ rpm. Velocity fluctuations from the transient vortices are not observed in Figure 9(a) and also not in Figure 10(a). It is worth noting that streaks due to transient vortices were not transcribed on the oil-film of the disk at $N_{\mathrm{o}} \geq \pm 300 \mathrm{rpm}$ in our previous experiments [7]. The transient vortices are not also observed in the smoke-flow images (Figure 9(b) and Figure 10(b)). However, arc-shaped streaks similar to those in Figure 7(b) and Figure 8(b) are observed at $r / R<-0.5\left(r_{0} / R_{0}<0.5\right)$ in the case of $N_{0}=-300 \mathrm{rpm}$, and at $r / R>0.4 \quad\left(r_{\mathrm{o}} / R_{\mathrm{o}}>1.4\right)$ in the case of $N_{0}=+300 \mathrm{rpm}$, respectively. The arc-shaped streaks at $N_{0}= \pm 300 \mathrm{rpm}$ also move in the radially outward direction of the disk, which was determined from the visualization movies. The regions where the arcshaped streaks are observed in Figure 9(b) and Figure 10(b) are consistent with the region with high PSD in the low-frequency band in Figure 9(a) and Figure 10(a). Consequently, we attribute the arc-shaped streaks to lowfrequency disturbances.

\subsection{Characteristics of Traveling Vortices Caused by Low-Frequency Disturbances}

Figure 11(a) and Figure 11(b) show the probability density function of the radial traveling velocities of the lowfrequency disturbances and the intervals between low-frequency disturbances at $r / R=+0.9\left(r_{0} / R_{0}=1.9\right)$; 


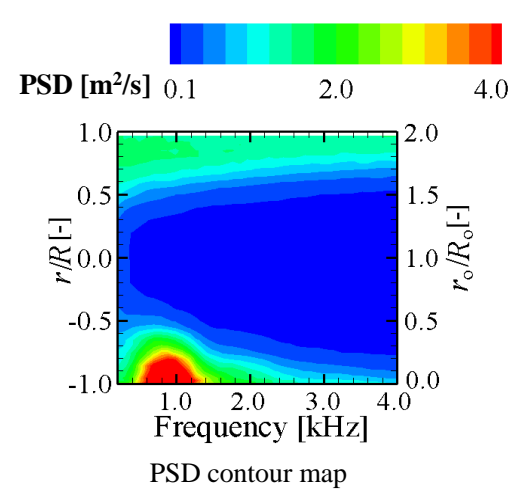

(a)

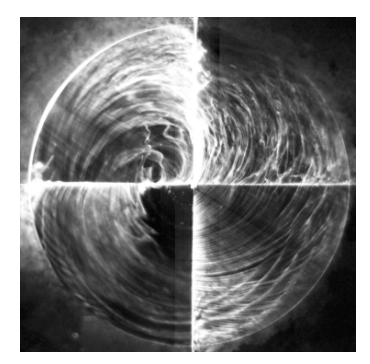

Visualization image

(b)

Figure 9. (a) Contour map of the ensemble-averaged PSD of $V_{\theta}^{*}$ fluctuation and (b) visualization image of top view with $N=+4000 \mathrm{rpm}, N_{\mathrm{o}}=$ $-300 \mathrm{rpm}$.

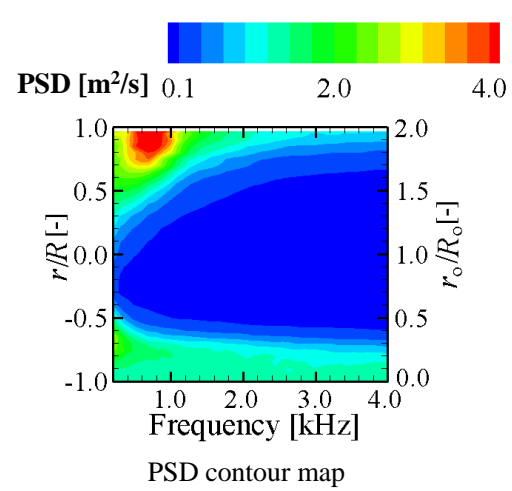

(a)

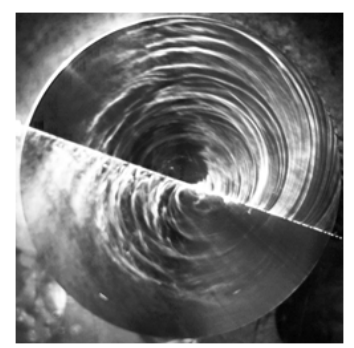

Visualization image

(b)

Figure 10. (a) Contour map of the ensemble-averaged PSD of $V_{\theta}^{*}$ fluctuation and (b) visualization image of the top view at $N=+4000 \mathrm{rpm}, N_{\mathrm{o}}=$ $+300 \mathrm{rpm}$.

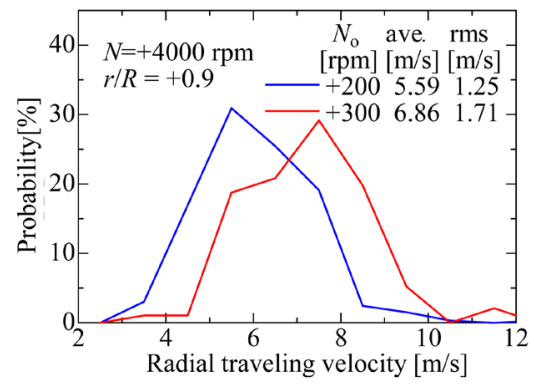

(a)

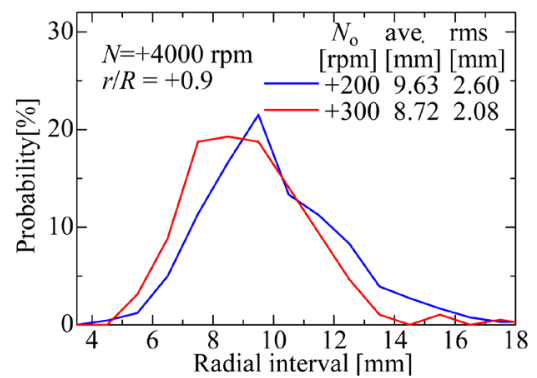

(b)

Figure 11. Characteristics of low-frequency disturbances. (a) Probability density function of the radial traveling velocities of the low-frequency disturbances (bin width: $1.0 \mathrm{~m} / \mathrm{s}$ ); (b) Probability density function of the radial intervals of the low-frequency disturbances (bin width: $1.0 \mathrm{~mm}$ ).

these were estimated from measurement data obtained by hot-wire probe. The radial traveling velocities and the intervals are consistent in the movies of the top view of the disk. Therefore, the arc-shaped streaks correspond to low-frequency disturbances generated by fluctuations in tangential velocity. The radial traveling velocities tend to increase with increasing orbital speed. In contrast, the intervals tend to decrease with increasing orbital speed. One possible reason for this is that the radial velocities increase with increasing orbital speed. 


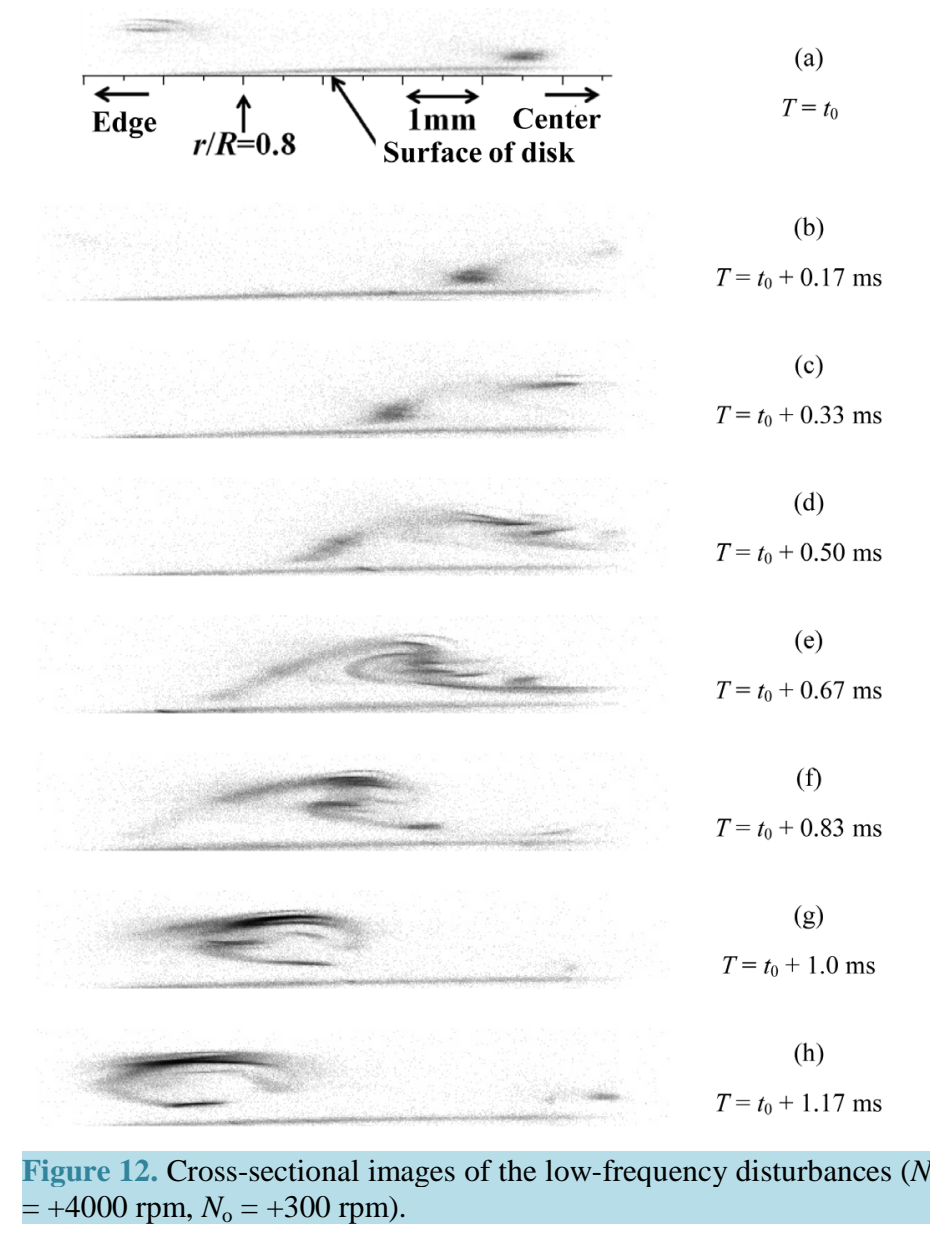

Figure 12 shows the cross-sectional structure of the low-frequency disturbances at $N=+4000 \mathrm{rpm}, N_{\mathrm{o}}=+300$ rpm. The figure is shown in negative, so the black areas are smoke. Fluid moving at high speed in the radially outward direction flows under fluid moving at low speed in the radially outward direction, and then fluid moving at low-speed is lifted up, as shown in Figure 12(d) and Figure 12(e). The lifted low-speed fluid moves to behind the high-speed fluid. The vortex structure of low-frequency disturbances is formed in this way, and the vortex structure changes while traveling in the radially outward direction.

\section{Conclusions}

The visualization images created by the smoke-wire method show that the behavior predicted for transient vortices under orbital motion of no more than $200 \mathrm{rpm}$ by using the PSD contour map of $V_{\theta}^{*}$ fluctuation agrees with observation. These vortices were not stationary relative to the rotational disk, and the intervals between the transient vortices became narrower in the increased-velocity region and became wider in the decreased-velocity region. At orbital speeds of $300 \mathrm{rpm}$ and faster, no transient vortices were seen in the visualization images.

The characteristics of low-frequency disturbances were shown by flow visualization and hot-wire measurements. It was found that the arc-shaped streaks on the disk correspond to low-frequency disturbances. The radial traveling velocities of low-frequency disturbances tended to increase and the intervals between them tended to decrease with increasing orbital speed. It was thus shown that the formation of the vortex structure of the lowfrequency disturbances, as well as the structure itself, changed while traveling in the radially outward direction.

\section{Acknowledgements}

This study was supported by a Grant-in-Aid for Scientific Research (No. 24560202) from the Japan Society for the Promotion of Science. 


\section{References}

[1] Reed, H.L. and Saric, W.S. (1989) Stability of Three Dimensional Boundary Layers. Annual Review of Fluid Mechanics, 21, 235-284. http://dx.doi.org/10.1146/annurev.fl.21.010189.001315

[2] Gregory, N., Stuart, J.T. and Walker, W.S. (1955) On the Stability of Three-Dimensional Boundary Layers with Application to the Flow Due to a Rotating Disk. Philosophical Transactions of the Royal Society of London, A248, 155199. http://dx.doi.org/10.1098/rsta.1955.0013

[3] Kohama, Y. (1984) Study on Boundary Layer Transition of a Rotating Disk. Acta Mechanica, 50, 193-199. http://dx.doi.org/10.1007/BF01170959

[4] Schlichting, H. (1955) Boundary-Layer Theory. McGraw-Hill, New York, 83-89.

[5] Von Kármán, T. (1921) Über laminare und turbulnte Reibung. Zeitschrift für Angewandte Mathematik und Mechanik, 1, 233-252. http://dx.doi.org/10.1002/zamm.19210010401

[6] Kobayashi, R., Kohama, Y. and Takamadate, Ch. (1980) Spiral Vortices in Boundary Layer Transition Regime on a Rotating Disk. Acta Mechanica, 35, 71-82. http://dx.doi.org/10.1007/BF01190058

[7] Munekata, M., Jobi, N., Ikebe, K. and Yoshikawa, H. (2012) Effects of Orbital Motion on the Boundary Layer Flow on a Spinning Disk. Open Journal of Fluid Dynamics, 2, 181-186. http://dx.doi.org/10.4236/ojfd.2012.24A020

[8] Munekata, M., Jobi, N., Kubo, K. and Yoshikawa, H. (2013) Characteristics of Transient Vortices in the Boundary Layer on a Rotating Disk under Orbital Motion. Journal of Thermal Science, 22, 600-605. http://dx.doi.org/10.1007/s11630-013-0668-0

[9] Malik, M.R., Wilkinson, S.P. and Orszag, S.A. (1981) Instability and Transition in Rotating Disk Flow. AIAA Journal, 19, 1131-1138. http://dx.doi.org/10.2514/3.7849

\section{Nomenclature}

$r \quad$ distance from center of disk (mm);

$r_{\mathrm{o}} \quad$ distance from center of orbit (mm);

$N \quad$ rotational velocity $(\mathrm{rpm}=$ revolutions $/ \mathrm{min})$;

$N_{\mathrm{o}} \quad$ orbital velocity (rpm);

$R \quad$ disk radius ( $\mathrm{mm})$;

$R_{0} \quad$ orbital radius (mm);

$\mathrm{z} \quad$ height from the disk surface (mm).

\section{Greek Letters}

$\delta \quad$ thickness of boundary layer under pure rotation $=(v / \omega)^{0.5}(\mathrm{~mm})$;

$v \quad$ kinematic viscosity of fluid $\left(\mathrm{m}^{2} / \mathrm{s}\right)$;

$\omega \quad$ angler velocity of rotating disk (rad/s). 
Scientific Research Publishing (SCIRP) is one of the largest Open Access journal publishers. It is currently publishing more than 200 open access, online, peer-reviewed journals covering a wide range of academic disciplines. SCIRP serves the worldwide academic communities and contributes to the progress and application of science with its publication.

Other selected journals from SCIRP are listed as below. Submit your manuscript to us via either submit@scirp.org or Online Submission Portal.
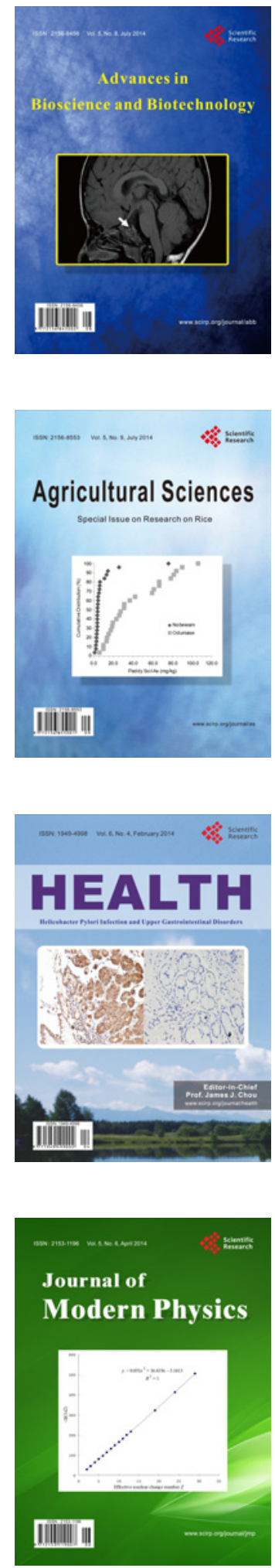
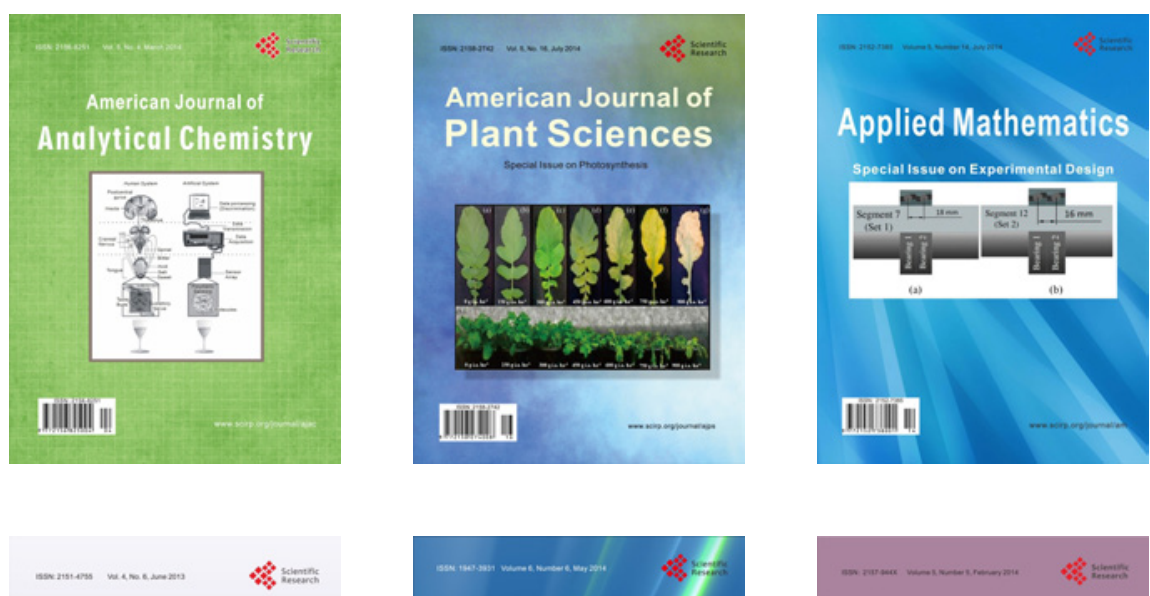

Creative Education
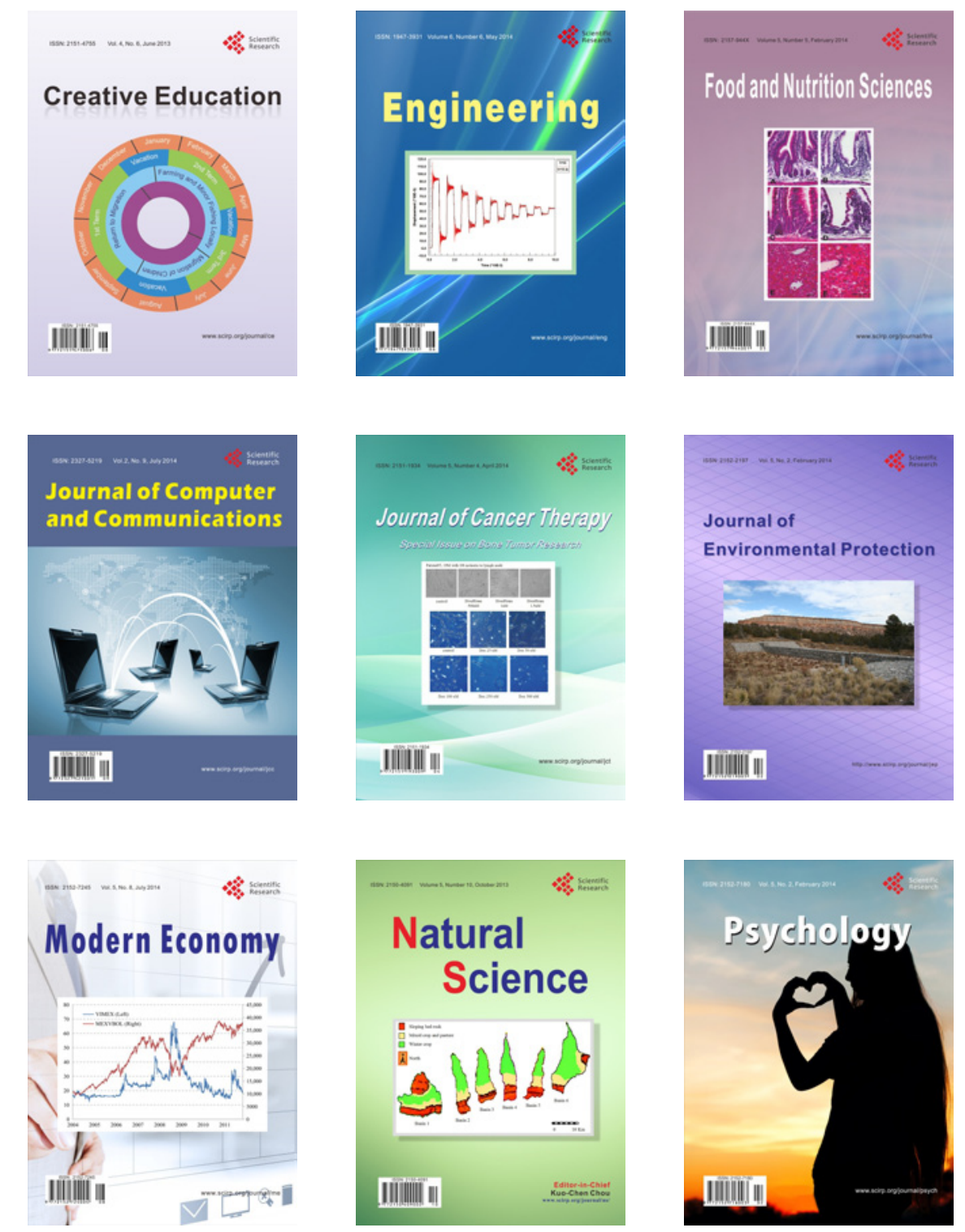